\title{
Author Correction: Clade-specific diversification dynamics of marine diatoms since the Jurassic
}

Eric Lewitus, Lucie Bittner (iD, Shruti Malviya, Chris Bowler and Hélène Morlon

Correction to: Nature Ecology and Evolution https://doi.org/10.1038/s41559-018-0691-3, published online 22 October 2018.

In the version of this Article originally published, the authors did not give credit to David G. Mann for the four microscopic images used in Fig. 1a. This has now been amended in all versions of the Article.

Published online: 13 November 2018

https://doi.org/10.1038/s41559-018-0740-y 